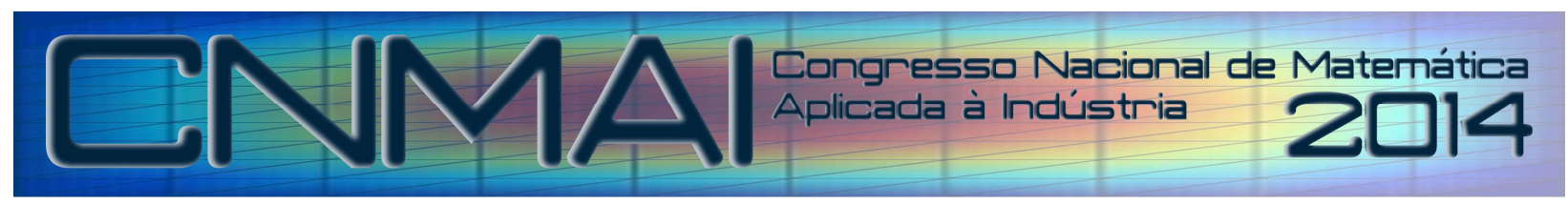

18 a 21 de novembro de 2014, Caldas Novas - Goiás

\title{
APLICAÇÃO DE TESTES DE REPETITIVIDADE E REPRODUTIVIDADE (R\&R) PARA VERIFICAÇÃO DE SCANNER LASER 3D
}

\author{
Antonio Piratelli-Filho, pirateli@unb.br ${ }^{1}$ \\ Laura Daniela Ordierez Zúñiga, lordierez@unb.br ${ }^{1}$ \\ Rosenda Valdés Arencibia, arvaldes@mecanica.ufu.br ${ }^{2}$
}

${ }^{1}$ Universidade de Brasília, UnB, Faculdade de Tecnologia, Depto. Engenharia Mecânica, 70910-900 Brasília, DF ${ }^{2}$ Universidade Federal de Uberlândia, UFU, Faculdade de Engenharia Mecânica, Uberlândia, MG

Resumo: $O$ objetivo deste trabalho é avaliar a aplicação de testes de Repetitividade e Reprodutividade (R\&R) para verificação de um Scanner Laser 3D. O teste aplicado envolveu um planejamento de experimento hierárquico com duas variáveis e execução da análise de variância (ANOVA) para verificar os efeitos e determinar um índice GRR. Esta verificação foi feita usando um padrão calibrado com diferentes elementos geométricos, tal como círculos, planos, esferas, etc. Os resultados desta análise foram comparados com a análise gráfica do desempenho do instrumento.

Palavras-chave: Repetitividade \& reprodutividade, teste de desempenho, laser scanner

\section{INTRODUÇÃO}

A variabilidade apresentada pelos instrumentos durante os procedimentos de medição pode interferir diretamente no resultado de uma inspeção na indústria, uma vez que pode proporcionar a aceitação de um produto fora de especificação ou rejeitar um produto dentro das especificações. Estas avaliações incorretas acarretam em custos extra de produção, seja pelo retrabalho para recuperar a peça ou pela perda de material e tempo de processamento. Uma forma de reduzir seu efeito na inspeção é através da seleção de um instrumento que apresente uma variabilidade ou uma incerteza de medição menor que a tolerância da peça, de forma que mesmo ainda havendo dúvida em relação ao resultado, ela seja minimizada a um custo aceitável do ponto de vista da empresa (Gonçalves-Júnior e Sousa, 2008).

A avaliação da variabilidade pode ser feita através de uma calibração. Nesta, deve ser adotado um padrão calibrado e com incerteza de medição conhecida, o qual deve ser medido repetidas vezes com o instrumento. Os erros sistemáticos e aleatórios deste sistema de medição são determinados e a incerteza de medição deve ser determinada segundo o Guia para Expressão da Incerteza de Medição (ISO TAG 4/WG 3, 2008). Embora esta referência esteja bem difundida no meio industrial, existem casos onde sua aplicação apresenta dificuldade, caso de instrumentos complexos cuja calibração é demorada e cara para ser realizada na empresa. Nestas situações, podem ser aplicados estudos de repetitividade e reprodutividade, chamados de R\&R, para avaliar a capacidade do sistema de medição quanto aos erros apresentados e classificados desta forma (AIAG, 2010; Peruchi et al., 2014).

Os parâmetros repetitividade e reprodutividade encontram suas definições no Vocabulário de Termos Gerais e Fundamentais em Metrologia (VIM), difundido no Brasil pelo Instituto Nacional de Metrologia, Qualidade e Tecnologia, INMETRO. A repetitividade dos resultados de medições consiste na capacidade do instrumento fornecer indicações muito similares ou parecidas, após medições sucessivas e repetidas, nas mesmas condições ambientais e de operação e num curto intervalo de tempo. A reprodutividade consiste no grau de concordância dos resultados de medições sucessivas de um mesmo mensurando, variando as condições de medição, tal como o princípio, o método, o observador, o padrão de referência, a localização, as condições de utilização e o tempo (INMETRO, 2012).

A determinação dos parâmetros nos estudos de repetitividade e reprodutividade $(R \& R)$ é baseada na análise estatística da dispersão dos resultados. Assim, a partir de um conjunto de resultados da medição de um padrão de referência, deve ser determinada a média, a amplitude, o desvio padrão e a variância, para caracterizar o desempenho do instrumento. A experimentação e a análise dos resultados devem ser executadas para separar os efeitos correspondentes à repetitividade e à reprodutividade e os métodos de análise mais empregados são: i) amplitude; ii) média e amplitude; iii) análise de variância (ANOVA); iv) análise de variância multivariada (MANOVA) (AIAG, 2010; Peruchi et al., 2014). 
O método da amplitude propõe a análise pela variabilidade total ou máxima, caracterizada pela amplitude dos erros determinados na medição do padrão. Neste método, adquire-se informação sobre a dispersão máxima dos erros do sistema de medição, porém não é possível especificar a repetitividade e a reprodutividade. Isto pode ser feito pelo método da média e amplitude, o qual ainda não permite identificar as interações entre fontes de repetitividade e reprodutividade, como efeito da interação do instrumento e operador. O método da análise de variância (ANOVA) permite analisar o erro de medição e determinar a influência das fontes de variabilidade presentes, separando e quantificando a dispersão proveniente da repetitividade e da reprodutividade, apresentados como um desvio padrão ou variância. A análise multivariada de variância (MANOVA) permite estudar a influência de muitas variáveis no processo de medição, sendo a análise conduzida pela ANOVA (AIAG, 2010; Peruchi et al., 2014).

O método da análise de variância tem sido utilizado para análise de sistemas de medição em estudos univariados, ou seja, quando somente o valor de uma variável resposta é determinado. Neste método, a variação observada pelos erros de medição pode ser decomposta em diferentes categorias ou ainda atribuída a diferentes variáveis envolvidas no processo de medição. Desta forma, planejando adequadamente o experimento, pode-se determinar o efeito de fatores responsáveis pela repetitividade e pela reprodutividade, como por exemplo efeito das condições ambientais, procedimento de medição, operador e do próprio instrumento. Na etapa de preparo, pode ser empregado um planejamento de experimentos como fatorial, fatorial fracionário ou hierárquico, dentre outros descritos pela literatura. Os resultados da aplicação deste planejamento são então analisados através da ANOVA, determinando os efeitos que influenciam significativamente a variabilidade (Box, Hunter e Hunter, 2005).

A ANOVA é aplicada através da decomposição da variabilidade total de um conjunto de resultados em parcelas associadas às fontes de variação investigadas. Isto é feito através das somas de quadrados dos desvios em relação à média (SQ), sendo determinadas uma SQ para cada variável ou fonte de variação (FV). A partir das SQs e do número de graus de liberdade associado às fontes de variação (GL), devem ser determinadas estimativas das variâncias de cada FV, através dos quadrados médios (QM) calculados pela razão entre os respectivos SQ e GL. A verificação da significância de cada FV é feita pela determinação da variável F, razão entre os QMs de cada fonte de variação e o QM do resíduo. No caso da análise de sistemas de medição, pode-se agrupar as FVs associadas à repetitividade e as FVs associadas à reprodutividade, de modo a estimar as variâncias de cada um e executar um teste comparativo. Os resultados são apresentados em uma tabela, incluindo uma comparação dos valores $\mathrm{F}$ calculados e os respectivos valores $\mathrm{F}$ esperados e determinados em tabelas da distribuição da estatística (Box, Hunter e Hunter, 2005). A tabela 1 apresenta o quadro de ANOVA para o caso geral.

Tabela 1. Quadro para determinação da ANOVA.

\begin{tabular}{ccccc}
\hline $\begin{array}{c}\text { Fonte de variação } \\
(\mathrm{FV})\end{array}$ & Soma de Quadrados & Grau de liberdade & Quadrado médio & F calculado \\
\hline Repetitividade & $(\mathrm{SQ})$ & $(\mathrm{GL})$ & $(\mathrm{QM})$ & \\
Reprodutividade & $\mathrm{SQ}$ repet & $\mathrm{GL}_{\text {repet }}$ & $\mathrm{SQ}_{\text {repet }} / \mathrm{GL}_{\text {repet }}$ & $\mathrm{QM}_{\text {repet }} / \mathrm{QM}_{\text {reprod }}$ \\
Total & $\mathrm{SQ}$ & $\mathrm{GL}_{\text {reprod }}$ & $\mathrm{SQ}_{\text {reprod }} / \mathrm{GL}_{\text {reprod }}$ & \\
\hline
\end{tabular}

Em estudos de repetitividade e reprodutividade (R\&R), a análise das variâncias determinadas é realizada conforme apresentado na equação 1, sendo que o valor GRR corresponde ao índice de R\&R, determinado em função das variâncias associadas à repetitividade e à reprodutividade. Analisando comparativamente, tem-se que se a reprodutividade for inferior a $10 \%$ da repetitividade, a reprodutividade é aceitável e pode-se afirmar que existe compatibilidade entre as diferentes condições adotadas. Se reprodutividade estiver entre $10 \%$ e $30 \%$ da repetitividade, considera-se que a reprodutividade entre as diferentes condições pode ser aceitável mas depende da importância do lote de peças solicitado, do custo dos instrumentos de medição, do custo da calibração ou reparação do instrumento, dentre outros. Se a reprodutividade for superior a 30\% da repetitividade, alguma ação deve ser tomada com relação ao sistema de medição, seja com respeito ao treinamento de pessoal, ao método empregado, às condições da medição ou até a aquisição de novo equipamento. Os dados obtidos na ANOVA podem auxiliar na tomada desta decisão (AIAG, 2010).

$$
G R R^{2}=\sigma_{\text {reprod }}^{2}+\sigma_{\text {repet }}^{2}
$$

Peruchi et al. (2014) realizaram uma revisão sobre os principais métodos de testes R\&R e relataram os trabalhos recentes publicados. Os autores destacaram as vantagens do método da ANOVA e suas aplicações e descreveram o uso da ANOVA multivariada, com aplicações. Dentre os principais, pode-se destacar trabalhos relacionados a ensaios em processos produtivos, processos automatizados de inspeção e ensaios da qualidade.

Atualmente não existe ainda um protocolo padrão para avaliar o desempenho de sistemas de imagem tridimensionais como scanners laser, bem como para avaliar os modelos CAD e sua geometria. Fabricantes de instrumentos estabelecem procedimentos de teste que minimizam o efeito da variação do ângulo de incidência nos erros sistemáticos, desconsiderando também os efeitos da profundidade (Beraldin et al., 2005). Gestel et al. (2009) desenvolveram um método empregando um artefato planar para verificar o efeito do ângulo de incidência e da profundidade de digitalização nos erros sistemáticos e aleatórios de um scanner laser 3D. Polo e Felicísimo (2012) verificaram a repetitividade de um scanner laser NextEngine, comparando as medições feitas em um objeto de forma complexa com os resultados da medição 
com um Paquímetro. Guidi et al. (2010) verificaram sete modelos de scanners 3D, determinando a resolução, a exatidão e a incerteza expandida, sendo que para um scanner NextEngine foram determinadas as incertezas expandidas (95\%) de $0,11^{\circ}$ para ângulos, $221 \mu \mathrm{m}$ para alturas e $12 \mu \mathrm{m}$ para raios. Barbero e Ureta (2011) realizaram um estudo comparando diferentes técnicas de digitalização, como tomografia computadorizada, MMC com sonda laser, scanner laser HandyScan, Scanner laser Krypton, dentre outros. Crescenzio et al. (2011) realizaram uma análise experimental para a compensação de erros sistemáticos de pontos XYZ obtidos por triangulação laser, usando um scanner laser Konica Minolta, variando a distância entre o objeto e o scanner e medindo um plano de referência e diferentes objetos calibrados (esfera, bloco e cilindros).

O objetivo deste trabalho é avaliar a aplicação de testes de Repetitividade e Reprodutividade (R\&R) para verificação de um Scanner Laser 3D. O teste aplicado envolveu um planejamento de experimento hierárquico com duas variáveis e execução da análise de variância (ANOVA) para verificar os efeitos e determinar um índice GRR. Esta verificação foi feita usando um padrão calibrado com diferentes elementos geométricos, tal como círculos, planos, esferas, etc. Os resultados desta análise foram comparados com a análise gráfica do desempenho do instrumento.

\section{METODOLOGIA}

O desenvolvimento deste trabalho foi feito usando um scanner laser 3D do fabricante NextEngine, modelo 2020i, o qual possui quatro fontes de feixes laser com $0,650 \mu \mathrm{m}$ de comprimento de onda, com uma potência de $10 \mathrm{~mW}$. A velocidade de digitalização é de 50000 pontos por segundo. Os modos disponíveis do scanner são Macro e Wide, sendo que a resolução e exatidão dimensional são 200 dpi e 0,127 mm para o modo Macro e 75 dpi e 0,381 mm para o modo Wide, respectivamente. O controle das etapas de digitalização foi feito com o software ScanStudio HD. A Figura 1 apresenta este instrumento, com a unidade emissora e receptora do feixe laser (câmera CCD) e a base para posicionamento da peça a digitalizar.

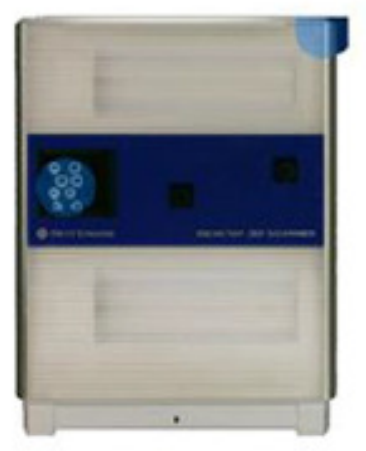

(A)

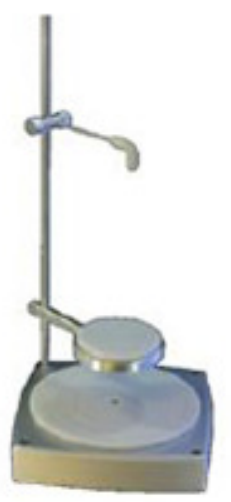

(B)

\section{Figura 1 - Scanner Laser 3D, com unidade de emissão e recepção do feixe laser (A) e base para posicionamento da peça sob medição.}

O scanner laser 3D foi empregado para digitalizar uma peça padrão desenvolvida em laboratório de fabricação, especialmente para servir de referência nos testes com o instrumento. Esta peça foi projetada para ter diferentes figuras geométricas, como esfera, círculos externo e interno (furo), retângulos externo e interno (furo), cone, cilindro, planos, geometria de forma irregular e escadas circular e irregular. A Figura 2 apresenta esta peça padrão, já com recobrimento para digitalização com o scanner. As dimensões dos elementos geométricos foram determinadas em uma Máquina de Medir por Coordenadas (MMC) modelo Cantilever, fabricada pela Mitutoyo, com incerteza expandida de medição em função do comprimento $\mathrm{L}(\mathrm{mm})$ de: $1,2+\mathrm{L} / 600 \mu \mathrm{m}$. 


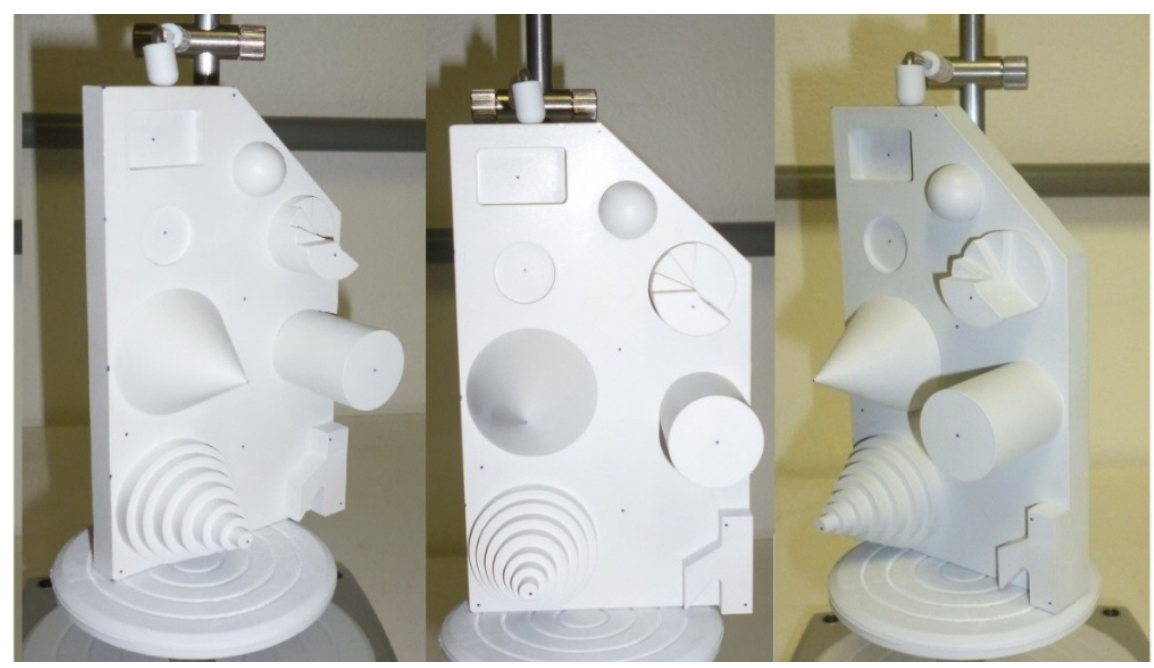

Figura 2 - Peça padrão empregada no teste do Scanner Laser 3D, em três diferentes posições para digitalização.

A Figura 3 apresenta a sequência de etapas desde a digitalização com o scanner laser 3D até a determinação das dimensões dos elementos geométricos da peça padrão. Inicialmente, a peça foi recoberta com tinta branca fosca para evitar a reflexão excessiva do feixe laser na superfície metálica. Depois, foi colocada sobre a base do scanner e fixada rigidamente com os apoios da base. Os parâmetros de digitalização foram selecionados no programa ScanStudio HD, que controla a ação do scanner laser, sendo escolhido o posicionamento SINGLE, o modo de alta definição HD e opção WIDE. A digitalização foi executada em três diferentes orientações da peça, sendo os pontos resultantes alinhados pelo comando ALIGN e START/REFINE_ALIGNMENT, antes de serem exportados com o comando OUTPUT/RAPIDWORKS. O software RapidWorks foi empregado para determinar as dimensões dos elementos geométricos, a partir das nuvens de pontos digitalizadas. Inicialmente, uma malha foi criada a partir da nuvem de pontos exportada, baseada nos métodos dos diagramas de Voronoi e da triangulação de Delaunay, usando o comando TOOLS/SCAN_TOOLS/MESH_BUILDUP_WIZARD/APPLY. A seguir, foi feita a classificação automática das regiões da malha obtida em diferentes geometrias regulares e conhecidas como planos, círculos e cilindros, usando o comando AUTOSEGMENT (REGION GROUP > AUTOSEGMENT > APPLY). O ajuste de pontos em locais específicos como a base do cilindro ou cone é feito adotando uma tolerância entre a malha e a superfície do sólido ajustado, a qual foi fixada em $0,1 \mathrm{~mm}$. As medidas das alturas foram feitas selecionando os planos inferior e superior e para as medidas de comprimentos foram selecionados os planos que se interceptam. Os valores determinados foram então comparados com os valores determinados na MMC, encontrando os erros de cada geometria digitalizada pela diferença do valor medido (scanner) menos o valor padrão (MMC). Os valores de média e desvio padrão dos erros foram determinados a partir dos resultados de três medições realizadas.

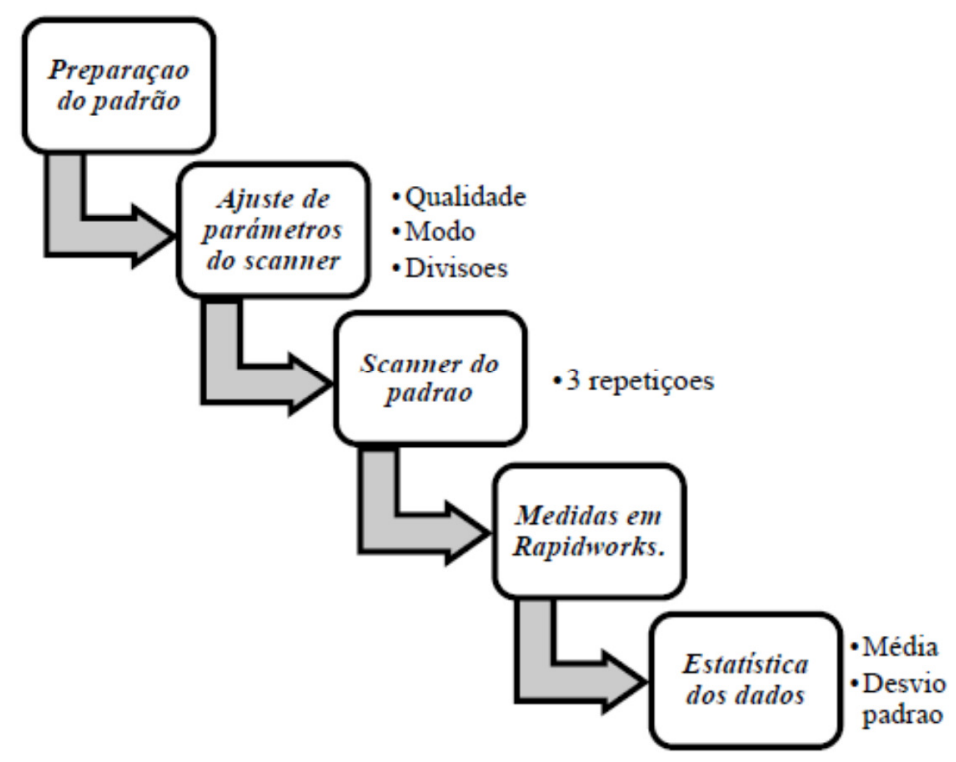

Figura 3 - Sequência de etapas de medição e análise. 
Com o intuito de avaliar a repetitividade e reprodutividade, um experimento foi planejado para verificar os efeitos da geometria, dos parâmetros e da repetição da medição nos valores de erros da digitalização. Um planejamento hierárquico foi escolhido para atribuir estas três variáveis, chamadas de Geometria $(\mathrm{G})$, Parâmetro (P) e Medições (M), conforme mostrado na Figura 4. Foram adotadas 6 geometrias (cone, cilindro, furo circular, escada circular, escada irregular e furo retangular), dois parâmetros (diâmetro e altura) e 3 medições (réplicas). As variáveis geometria e parâmetro estão associadas à repetitividade e a variável medições à reprodutividade, uma vez que foram executadas em dias e condições ambientais diferentes. A análise dos erros encontrados foi executada pela técnica de Análise de Variância (ANOVA), sendo que o quadro de cálculo está apresentado na Tabela 2. Nesta tabela, os valores dos quadrados médios das variáveis são determinados pelas expressões (2), (3) e (4).
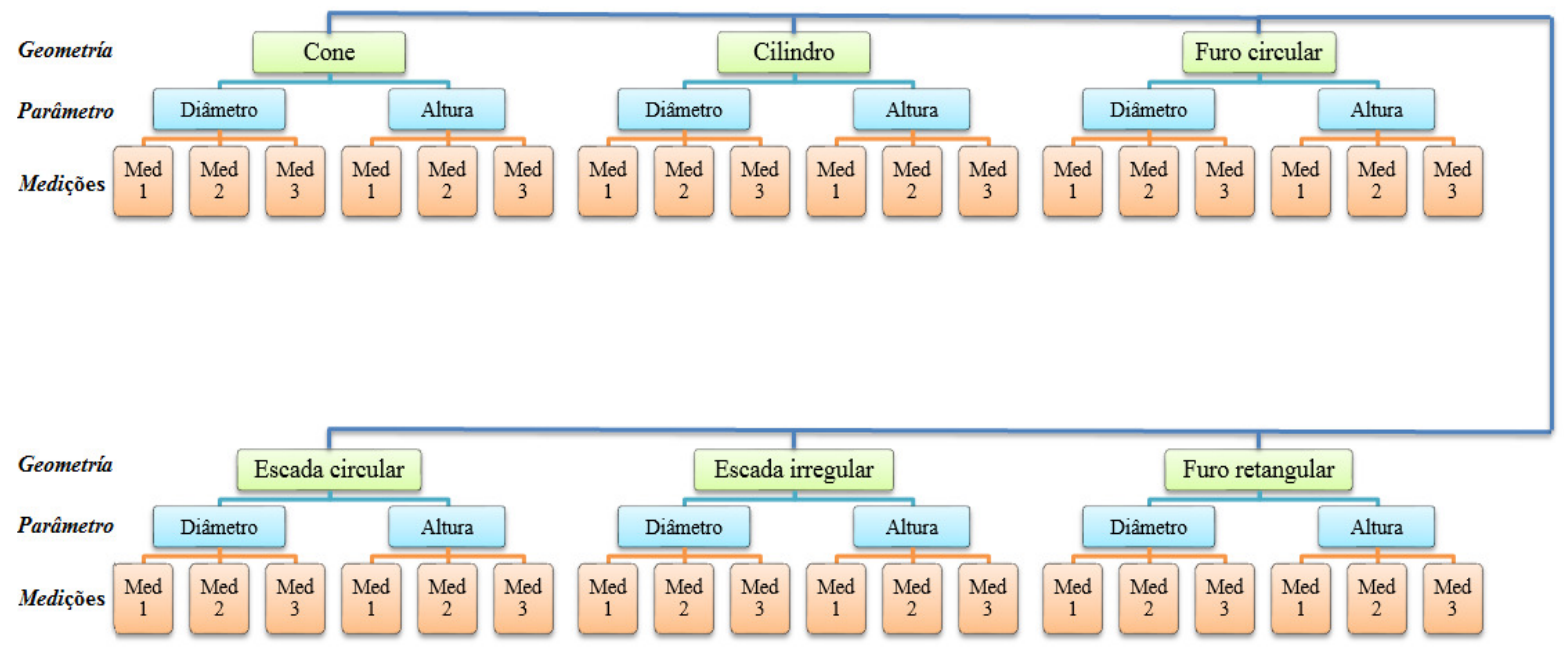

Figura 4 - Variáveis e níveis adotados no planejamento hierárquico.

Tabela 2 - Quadro de Análise de Variância para o planejamento hierárquico.

\begin{tabular}{|c|c|c|c|c|}
\hline $\begin{array}{l}\text { Fonte de } \\
\text { variação }\end{array}$ & Soma de quadrados SQ & $\begin{array}{c}\text { Graus de } \\
\text { liberdade } \\
\text { GL }\end{array}$ & $\begin{array}{c}\text { Quadrados } \\
\text { médios } \\
\text { QM }\end{array}$ & $\begin{array}{l}\text { Valor esperado dos } \\
\text { quadrados médios }\end{array}$ \\
\hline Média & G.P.M. $\bar{y}^{2}$ & 1 & - & - \\
\hline Geometrias & $-\bar{y})^{2}$ & $G-1$ & QMG & $P . M . \sigma_{g}^{2}+M . \sigma_{p}^{2}+\sigma_{m}^{2}$ \\
\hline Parâmetros & $\left.p-\bar{y}_{g}\right)^{2}$ & $G *(P-1)$ & QMP & $M \cdot \sigma_{p}^{2}+\sigma_{m}^{2}$ \\
\hline Medições & & $G * P *(M-1)$ & QMM & $\sigma_{m}^{2}$ \\
\hline Total & $\sum_{p} \sum_{m}\left(\bar{y}_{g p m}\right)$ & $G * P * M$ & - & - \\
\hline
\end{tabular}

$$
\begin{gathered}
Q M G=\frac{P M \sum_{g}^{G}\left(\bar{y}_{g}-\bar{y}\right)^{2}}{G-1} \\
Q M P=\frac{M \sum_{g}^{G} \sum_{p}^{P}\left(\bar{y}_{g p}-\bar{y}_{g}\right)^{2}}{G(P-1)} \\
Q M M=\frac{\sum_{g}^{G} \sum_{p}^{P} \sum_{m}^{M}\left(\bar{y}_{g p m}-\bar{y}_{g p}\right)^{2}}{n-1}
\end{gathered}
$$




\section{RESULTADOS}

Os resultados com os erros de medição das geometrias da peça padrão estão apresentados na Tabela 3. A Tabela 4 apresenta os resultados da ANOVA. A partir destes resultados, observa-se que os quadrados médios da geometria apresentaram o maior valor, seguido dos quadrados médios dos parâmetros e da medição. Isto permite afirmar que a variabilidade associada a geometria tem um efeito maior na variabilidade dos erros de medição com o scanner laser 3D utilizado, em relação ao parâmetro medido e à medição.

Tabela 3 - Erros de digitalização associados às variáveis investigadas no planejamento hierárquico.

\begin{tabular}{|l|c|c|c|c|c|c|c|}
\hline & & \multicolumn{6}{|c|}{ Geometria } \\
\hline Parâmetro & Medição & Cone & Cilindro & $\begin{array}{c}\text { Furo } \\
\text { circular }\end{array}$ & $\begin{array}{c}\text { Escada } \\
\text { circular }\end{array}$ & $\begin{array}{c}\text { Escada } \\
\text { irregular }\end{array}$ & $\begin{array}{c}\text { Furo } \\
\text { retangular }\end{array}$ \\
\hline \multirow{3}{*}{ Diâmetro } & $\mathbf{1}$ & 0,571 & 0,365 & $-0,563$ & 0,117 & 0,316 & 0,040 \\
\cline { 2 - 8 } & $\mathbf{2}$ & 0,651 & 0,534 & $-0,571$ & 0,065 & 1,052 & 0,031 \\
\cline { 2 - 9 } & $\mathbf{3}$ & 0,689 & 0,510 & $-0,570$ & 0,147 & 0,212 & 0,101 \\
\hline \multirow{3}{*}{ Altura } & $\mathbf{1}$ & 0,326 & 0,208 & 0,015 & 0,124 & 0,408 & 0,645 \\
\cline { 2 - 9 } & $\mathbf{2}$ & 0,326 & 0,304 & 0,122 & 0,527 & 0,333 & 0,895 \\
\cline { 2 - 9 } & $\mathbf{3}$ & $-0,930$ & 0,338 & 0,084 & 0,096 & 0,442 & 0,584 \\
\hline
\end{tabular}

Tabela 4 - Resultados da ANOVA

\begin{tabular}{cccc}
\hline $\begin{array}{c}\text { Fonte de } \\
\text { variação }\end{array}$ & $\begin{array}{c}\text { Soma de quadrados } \\
\text { SQ }\end{array}$ & $\begin{array}{c}\text { Graus de } \\
\text { liberdade } \\
\text { GL }\end{array}$ & $\begin{array}{c}\text { Quadrados médios } \\
\text { QM }\end{array}$ \\
\hline Média & 468,3485 & 1 & - \\
Geometrias & 2,1993 & 5 & 0,4399 \\
Parâmetros & 1,7499 & 6 & 0,2916 \\
Medições & 1,0260 & 24 & 0,0428 \\
Total & 328,7196 & 36 & - \\
\hline
\end{tabular}

As Equações (5) a (8) apresentam o cálculo dos componentes de variância das variáveis medição, parâmetro e geometria, desenvolvidas a partir da Tabela 2. Estes cálculos permitem separar os efeitos acumulados no planejamento hierárquico, pois o efeito das medições está embutido nos efeitos dos parâmetros e da geometria. Assim, nota-se que a variância estimada dos parâmetros apresentou a maior influência nos erros do scanner, de 0,1244 mm², seguido pela variância das medições igual a $0,0428 \mathrm{~mm}^{2}$ e da geometria igual a $0,0040 \mathrm{~mm}^{2}$. Nota-se que neste planejamento, o efeito dos parâmetros estava incluído no efeito da geometria, no caso da ANOVA, demostrado pelos valores dos quadrados médios.

$$
\begin{gathered}
\sigma_{m}^{2}=Q M M=0,0428 \\
\sigma_{p}^{2}=\frac{\left(Q M P-\sigma_{m}^{2}\right)}{2}=0,1244 \\
\sigma_{g}^{2}=\frac{Q M G-3 \cdot \sigma_{p}^{2}-\sigma_{m}^{2}}{6}=0,0040
\end{gathered}
$$

O valor do índice de repetitividade e reprodutividade GRR foi determinado pela expressão 8 e foi igual a 0,414. Este índice foi considerado para representar a variabilidade do instrumento de medição. Uma análise separando a repetitividade da reprodutividade pode ser feira, considerando que a repetitividade pode ser associada à variabilidade dos parâmetros e da geometria e que a reprodutividade pode ser associada à variabilidade ocasionada pelas medições. Assim, temos que as variâncias podem ser determinadas como sendo $\sigma_{\text {repet }}^{2}=0,1284 \mathrm{~mm}^{2}$ e $\sigma_{\text {reprod }}^{2}=0,0428 \mathrm{~mm}^{2}$.

$$
G R R=\sqrt{\sigma_{g}^{2}+\sigma_{p}^{2}+\sigma_{m}^{2}}=0,414
$$

Os resultados do teste apresentado foram comparados com o teste R\&R feito pela análise da média e amplitude, método da análise gráfica. As Figuras 5 e 6 apresentam os resultados do teste R\&R considerando a análise gráfica, para os erros na medição das alturas e dos diâmetros, respectivamente. Os intervalos de confiança foram determinados a partir do desvio padrão das medições, adotando uma confiança de $95 \%$ e 2 graus de liberdade, sendo $t=4,303$. As linhas azuis escuras representam o valor do erro máximo admitido pelo fabricante do instrumento, chamado de exatidão, igual a 0,381 mm (modo Wide do scanner laser). A definição do desempenho pode ser feita pela amplitude da média dos desvios 
encontrados, tanto para as medidas de altura como para as medidas de diâmetro. Assim, os valores estabelecidos são 2,0 mm para altura e $0,8 \mathrm{~mm}$ para diâmetro.

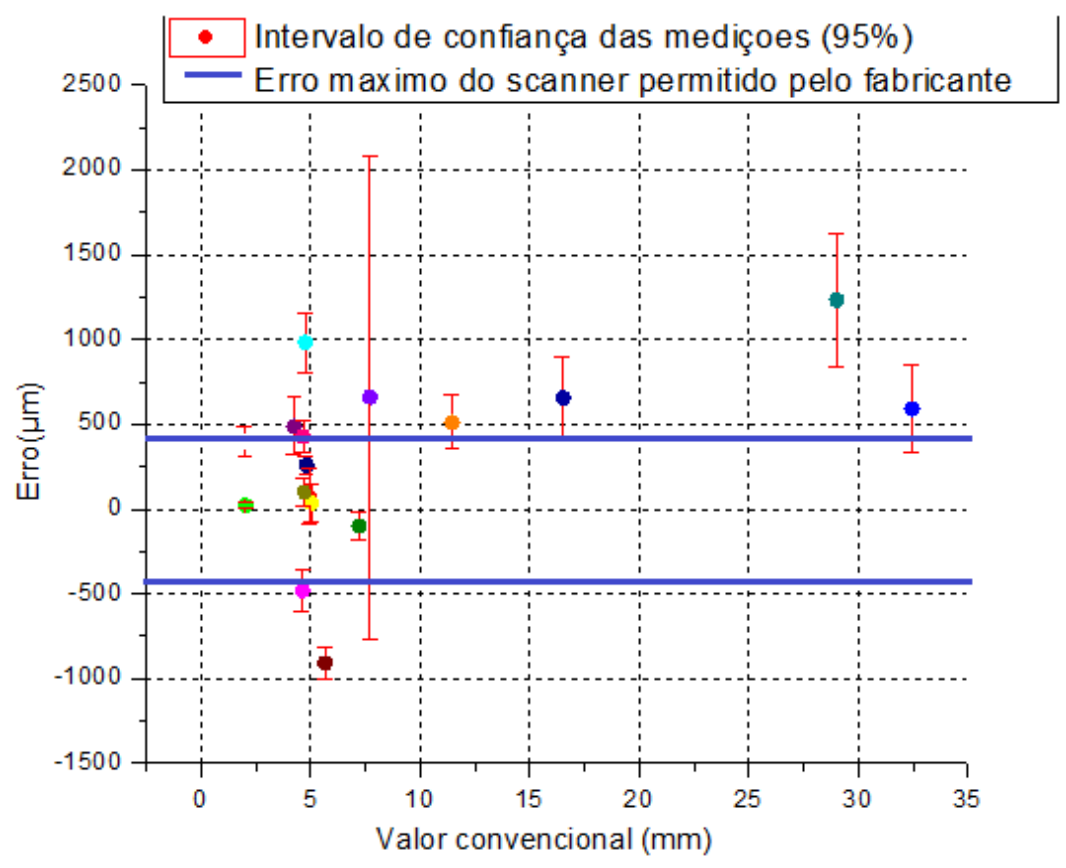

Figura 5. Valores dos erros em função das alturas na peça.

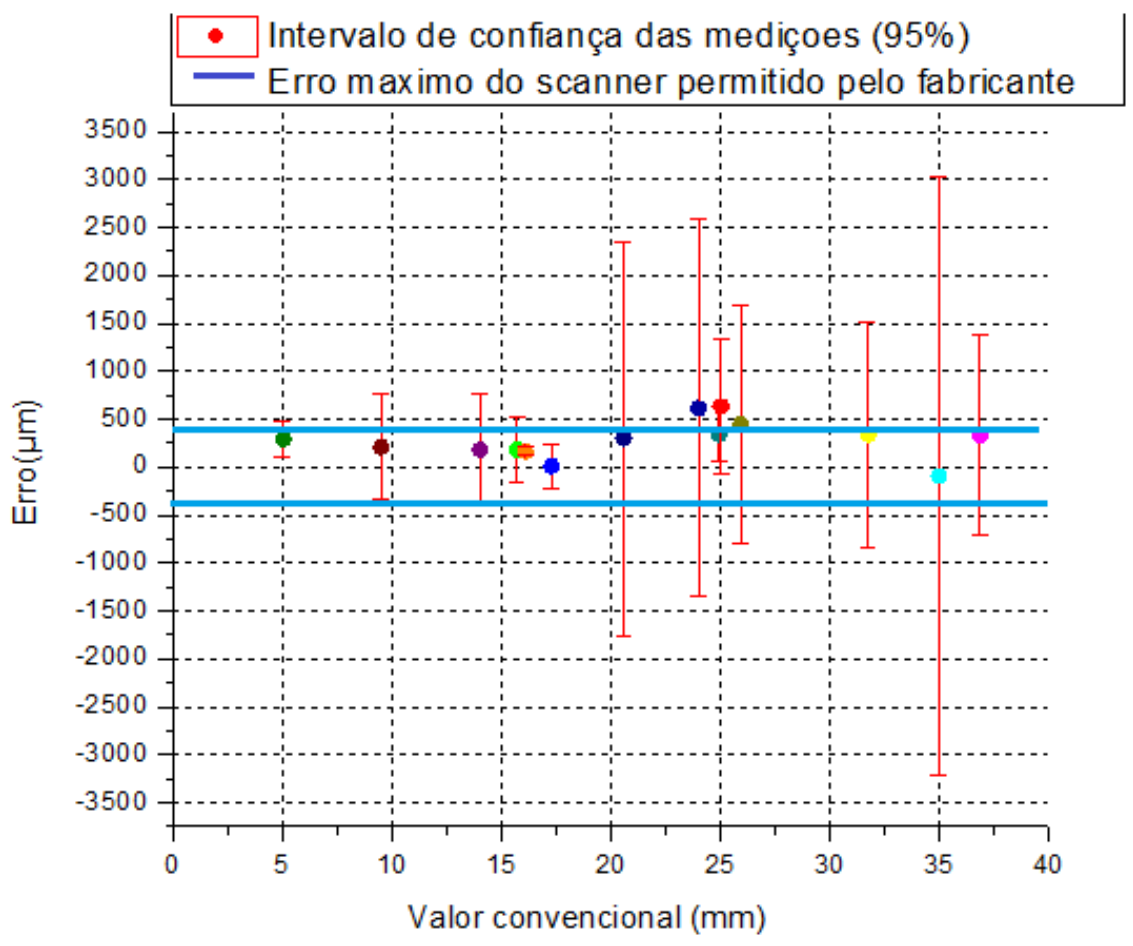

Figura 6. Valores dos erros em função dos diâmetros na peça.

\section{CONCLUSÕES}

Uma análise do desempenho de um scanner laser 3D foi apresentada através de um teste de repetitividade e reprodutividade (R\&R). O teste foi executado em um scanner laser NextEngine com o auxílio de um a peça padrão desenvolvida em alumínio. A peça foi calibrada em uma Máquina de Medir por Coordenadas (MMC) para determinação das alturas e diâmetros dos seus elementos geométricos. As medições com o scanner laser permitiram a determinação destas alturas e diâmetros, o que possibilitou o cálculo dos erros de medição. 
O teste R\&R proposto foi executado através da aplicação de um planejamento de experimentos hierárquico com três variáveis, a geometria, os parâmetros e as medições. A análise foi efetuada através da Análise de Variância (ANOVA) e as variâncias associadas às variáveis foram determinadas. O teste permitiu determinar as variâncias associadas à repetitividade e à reprodutividade, além de um índice $R \& R$, o que torna a análise mais completa que a verificação gráfica da amplitude dos erros médios proposta pelo método da análise gráfica.

Futuros trabalhos podem envolver a análise multivariada de variância, MANOVA, para analisar o desempenho de instrumentos como o scanner laser 3D ou outros, como já sugere a literatura sobre o assunto.

\title{
REFERÊNCIAS
}

AIAG. 2010. Measurement systems analysis: reference manual. 4th ed. Detroit, MI, USA: Automotive Industry Action Group.

Barbero, B.R., Ureta, E.S. 2011. Comparative study of different digitization techniques and their accuracy. ComputerAided Design. Vol. 43 (2). 188-206.

Beraldin, J.A., Gaiani, M. 2005. Evaluating the Performance of Close-Range 3D ActiveVision Systems for Industrial Design Applications. Videometrics VIII - SPIE Electronic Imaging. Vol. 5665. 67-77.

Box, G.E.P., Hunter, J.S., Hunter, W.G. 2005. Statistics for experimenters. $3^{\text {rd }}$ Edition. New Jersey, EUA: John Wiley \& Sons. 633 p.

Crescenzio, F., Fantini, M. 2011. Experimental analysis for error compensation of laser scanner data. Proc. International conference on Innovative Methods in Product Design, Venice, Italy.

Gestel, N.V., Cuypers, S., Bleys, P., Kruth, J.P. 2009. A performance evaluation test for laser line scanners on CMMs. Optics and Lasers in Engineering. Vol. 47 (3-4). 336-342.

Gonçalves-Júnior, A.A., Sousa, A.R. 2008. Fundamentos de Metrologia Científica e Industrial. São Paulo: Ed. Manole. $407 \mathrm{p}$.

Guidi, G., Russo, M., Magrassi, G., Bordegoni, M. 2010. Performance evaluation of triangulation based range sensors. Sensors. Vol. 10. 7192 -215.

ISO TAG 4/WG 3. 2008. Guide to the Expression of Uncertainty in Measurement. Geneva, Suíça. 141p.

INMETRO. 2012. Vocabulário Internacional de Metrologia: Conceitos Fundamentais e Gerais e Termos Associados. $1^{\mathrm{a}}$ edição Luso-brasileira. Rio de Janeiro. 95 p.

Peruchi, R.S., Paiva, A.P., Balestrassi, P.P., Ferreira, J.R., Sawhney, R. 2014. Weighted approach for multivariate analysis of variance in measurement system analysis. Precision Engineering. Vol. 38. 651-658.

Polo, M.E, Felicísimo, A.M. 2012. Analysis of uncertainty and repeatability of a low-cost 3D laser scanner. Sensors. Vol. 12 (7). $9046-9054$.

\section{RESPONSABILIDADE AUTORAL}

Os autores são os únicos responsáveis pelo conteúdo deste trabalho.

\section{APPLICATION OF REPEATABILITY AND REPRODUCIBILITY (R\&R) TESTS FOR LASER SCANNER 3D PERFORMANCE TESTS}

\author{
Antonio Piratelli-Filho, pirateli@unb.br ${ }^{1}$ \\ Laura Daniela Ordierez Zúñiga, lordierez@unb.br' ${ }^{1}$ \\ Rosenda Valdés Arencibia, arvaldes@mecanica.ufu.br ${ }^{2}$
}

${ }^{1}$ Universidade de Brasília, UnB, Faculdade de Tecnologia, Depto. Engenharia Mecânica, 70910-900 Brasília, DF
${ }^{2}$ Universidade Federal de Uberlândia, UFU, Faculdade de Engenharia Mecânica, Uberlândia, MG

Abstract. The objective of this study is to evaluate the application of Repeatability and Reproducibility tests $(R \& R)$ for verification of a $3 D$ Laser Scanner. The applied test involved the design of a hierarchical experiment with two variables and perform the analysis of variance (ANOVA) to investigate the effects and determining a GRR index. This verification was done using a calibrated gauge with different geometric elements, such as circles, planes, spheres, etc. The results of this analysis were compared with the graphical analysis of instrument performance.

Keywords: repeatability and reproducibility, performance tests, laser scanner 